\title{
Weak coupling limit of $2+1$ SU(2) LGT and mass gap.
}

\section{T. P. Sreeraj*}

The Institute of Mathematical Sciences, C.I.T. campus, Taramani, Chennai 600113, India

E-mail: sreerajtp@imsc.res.in, sreeraj.tp@gmail.com

\section{Ramesh Anishetty}

The Institute of Mathematical Sciences, C.I.T. campus, Taramani, Chennai 600113, India

E-mail: ramesha@imsc.res.in

Dual description of $S U(2)$ lattice gauge theory in $2+1$ dimensions is shown to be the theory of interacting gauge invariant 'abelian like' electric loops. The Gauss law is solved exactly to construct the Hilbert space of the gauge invariant theory using the Schwinger boson representation. This is achieved by envisaging what is called the 'splitting of a point'. Such a 'point split' lattice allows us to describe non local electric loops in terms of three independent, local, gauge invariant quantum numbers at each site satisfying triangle inequalities. The matrix elements of the Hamiltonian becomes simpler and triangle inequalities become subdominant in the weak coupling limit. The closed loop dynamics is analysed using a gauge invariant phase space path integral. In the weak coupling limit, the mean gauge invariant electric flux becomes large and small spatial electric flux loops dominate in the vacuum state leading to a mass gap.

The 36th Annual International Symposium on Lattice Field Theory - LATTICE2018

22-28 July, 2018

Michigan State University, East Lansing, Michigan, USA.

\footnotetext{
* Speaker.
} 


\section{Introduction}

There had been various attempts [1] in the past to remove the redundant gauge degrees of freedom by describing gauge theories in terms of gauge invariant Wilson loops. These attempts to construct a complete set of gauge invariant Wilson loop operators as well as loop states are difficult due to non local nature and overcompleteness of the set of all Wilson loops. In this paper, we will describe a complete, local, gauge invariant formulation of $\mathrm{SU}(2)$ lattice gauge theory (LGT) on what we call a 'point split lattice'[2].

\section{Hamiltonian formulation of $\mathrm{SU}(2)$ lattice gauge theory}

The basic variables of the Kogut Susskind formulation [4] of SU(2) LGT are the operator valued $2 \times 2 \mathrm{SU}(2)$ matrices $U_{i}(\vec{x})$ lying on the links starting from the site $\vec{x}$ along the $i$ direction, of a spatial square lattice and their conjugate left and right electric fields $E_{i}^{a}(\vec{x}), E_{\vec{i}}^{a}(\vec{x}+\hat{i})$. They satisfy the following commutation relation:

$$
\left[E_{i}^{a}(\vec{x}), U_{i}(\vec{x})\right]=-\left(\frac{\sigma^{a}}{2} U_{i}(\vec{x})\right) ; \quad\left[E_{\bar{i}}^{a}(\vec{x}+\hat{i}), U_{i}(\vec{x})\right]=\left(U_{i}(\vec{x}) \frac{\sigma^{a}}{2}\right)
$$

$\sigma^{a}$ are the Pauli matrices. $E_{i}^{a}, E_{\bar{i}}^{a}$ satisfy the $\mathrm{SU}(2)$ algebra. The right electric field is defined as the negative of the left electric field parallel transported along the link. I.e, $E_{\bar{i}}^{a}(\vec{x}+i)=R_{a b}(U) E_{i}^{a}(\vec{x})$, where $R_{a b}(U)=\frac{1}{2} \operatorname{Tr}\left(U \sigma^{a} U^{\dagger} \sigma^{b}\right)$. Therefore,

$$
E_{i}^{a}(\vec{x}) E_{i}^{a}(\vec{x})=E_{\dot{i}}^{a}(\vec{x}+\hat{i}) E_{\bar{i}}^{a}(\vec{x}+\hat{i})=E^{2}(\vec{x}) \quad \text { link constraint }
$$

The lattice electric fields are dimensionless and are related to the dimensionful continuum electric fields as $E_{\text {cont }} \sim a^{-d / 2} E_{L}$ where $d$ is the spacetime dimension and $a$ is the lattice spacing, so that the electric field components commute on continuum limit. The Hamiltonian is given by:

$$
H=g^{2} \sum_{\vec{x}, i} E_{i}^{a}(\vec{x}) E_{i}^{a}(\vec{x})+\frac{2}{g^{2} a^{2}} \sum_{p}\left[2-\operatorname{Tr} U_{p}\right]
$$

In (2.3), $p$ denotes plaquettes. The Hamiltonian along with the Gauss law constraint, $\mathscr{G}^{a}(\vec{x})=$ $\sum_{i=1}^{2}\left[E_{i}^{a}(\vec{x})+E_{\vec{i}}^{a}(\vec{x})\right]=0$, completely describes the theory. $\mathscr{G}^{a}(\vec{x})$ is the generator of gauge transformations at site $\vec{x}$.

\subsection{Prepotential formulation}

It is convenient to reformulate SU(2) LGT in terms of harmonic oscillator doublets [5] (Schwinger bosons) which tranform under the fundamental representation of the gauge group. Electric fields and the link operator can be written in terms of Schwinger bosons as

$$
\begin{gathered}
E_{i}^{a}(\vec{x}) \equiv a_{i, \alpha}^{\dagger}(\vec{x})\left(\frac{\sigma^{a}}{2}\right)_{\alpha \beta} a_{i, \beta}(\vec{x}), \quad E_{\bar{i}}^{a}(\vec{x}+\hat{i}) \equiv a_{\vec{i}, \alpha}^{\dagger}(\vec{x}+\hat{i})\left(\frac{\sigma^{a}}{2}\right)_{\alpha \beta} a_{\bar{i}, \beta}(\vec{x}+\hat{i}) . \\
\left(U_{i}\right)_{\alpha \beta}(\vec{x})=\frac{1}{\sqrt{\hat{N}+1}}\left[\tilde{a}_{i, \alpha}^{\dagger}(\vec{x}) a_{\vec{i}, \beta}^{\dagger}(\vec{x}+\hat{i})+a_{i, \alpha}(\vec{x}) \tilde{a}_{\bar{i}, \beta}(\vec{x}+\hat{i})\right] \frac{1}{\sqrt{\hat{N}+1}}
\end{gathered}
$$




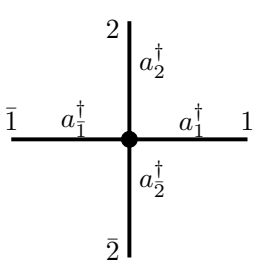

(a)

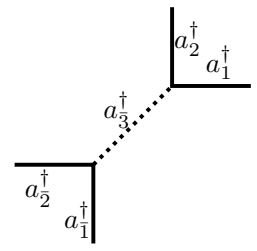

(b)

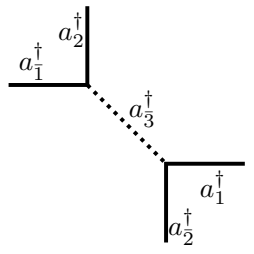

(c)

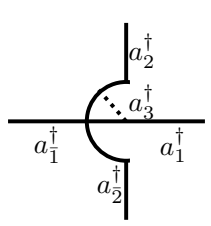

(d)

Figure 1: (a) Schwinger bosons in the original square lattice. (b),(c),(d) denotes three different splitting schemes at each vertex. Point splitting reduces a 4 vertex to two 3 -vertices.

Above, $a_{i, \alpha}^{\dagger}(\vec{x})$ and $a_{i, \alpha}^{\dagger}(\vec{x}+\hat{i})$ are the harmonic oscillator doublets at the left and right of the link operator $U_{i}(\vec{x}) . \tilde{a}_{\alpha}^{\dagger}=\varepsilon_{\alpha \gamma} a_{\gamma}^{\dagger}$ and the number operator $\hat{N} \equiv a_{i}^{\dagger}(\vec{x}) \cdot a_{i}(\vec{x})=a_{i}^{\dagger}(\vec{x}+\hat{i}) \cdot a_{\bar{i}}(\vec{x}+\hat{i})$ due to the link constraint (2.2). The number of Schwinger bosons at the left end of a link equals that at the right end. Schwinger bosons transform under gauge transformation $\Lambda$ at any site as $a_{\alpha} \stackrel{\Lambda}{\rightarrow} \Lambda_{\alpha \beta} a_{\beta}$. Note that $\tilde{a}_{\alpha}^{\dagger}$ transforms the same way as $a_{\alpha} . a_{i}^{\dagger}(\vec{x}) \cdot \tilde{a}_{i^{\prime}}^{\dagger}(\vec{x}) ; i \neq i^{\prime}$ is gauge invariant. There are 6 pairs of different Schwinger bosons at any site on a 2 dimensional square lattice. Therefore, a gauge invariant basis can be constructed as:

$\left|l_{12}, l_{1 \overline{1}}, l_{1 \overline{2}}, l_{2 \overline{1}}, l_{2 \overline{2}}, l_{\overline{1}} \bar{\nu}\right\rangle=\left(a_{1}^{\dagger} \cdot \tilde{a}_{2}^{\dagger}\right)^{l_{12}}\left(a_{1}^{\dagger} \cdot \tilde{a}_{\overline{1}}^{\dagger}\right)^{l_{1} \overline{1}}\left(a_{1}^{\dagger} \cdot \tilde{a}_{\overline{2}}^{\dagger}\right)^{l_{1} \overline{2}}\left(a_{2}^{\dagger} \cdot \tilde{a}_{\overline{1}}^{\dagger}\right)^{l_{2} \overline{1}}\left(a_{2}^{\dagger} \cdot \tilde{a}_{\overline{2}}^{\dagger}\right)^{l_{2} \overline{2}}\left(a_{\overline{1}}^{\dagger} \cdot \tilde{a}_{\overline{2}}^{\dagger}\right)^{l_{\overline{1}}}|0\rangle$

Above, $|0\rangle$ is the strong coupling vacuum. $E^{a}|0\rangle=a|0\rangle=0$. However, not all such states are independent due to the following relation ${ }^{1}:\left(a_{1}^{\dagger} \cdot \tilde{a}_{2}^{\dagger}\right)\left(a_{\overline{1}}^{\dagger} \cdot \tilde{a}_{\overline{2}}^{\dagger}\right)=\left(a_{1}^{\dagger} \cdot \tilde{a}_{\overline{1}}^{\dagger}\right)\left(a_{2}^{\dagger} \cdot \tilde{a}_{\overline{2}}^{\dagger}\right)+\left(a_{1}^{\dagger} \cdot \tilde{a}_{\overline{2}}^{\dagger}\right)\left(a_{2}^{\dagger} \cdot \tilde{a}_{\overline{1}}^{\dagger}\right)$ which follows from $\varepsilon_{\alpha \beta} \varepsilon_{\bar{\alpha} \bar{\beta}}=\delta_{\alpha \bar{\alpha}} \delta_{\beta \bar{\beta}}+\delta_{\alpha \bar{\beta}} \delta_{\beta \bar{\alpha}}$. In order to construct a complete basis, we modify the lattice by a process we call 'splitting of the point'.

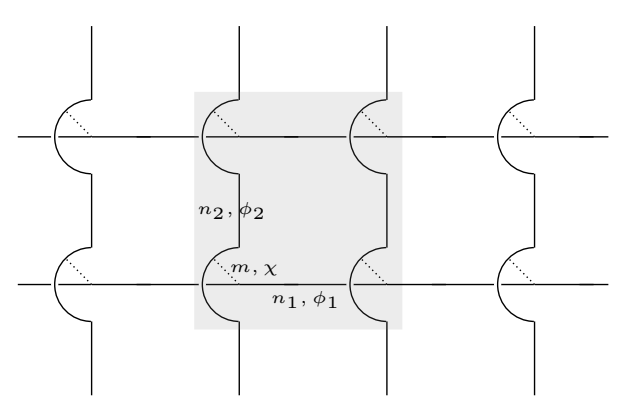

(a)

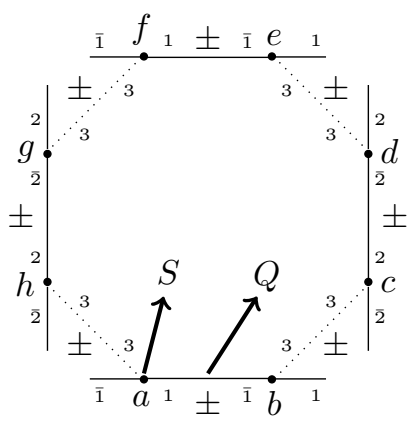

(b)

Figure 2: (a). The ps-lattice obtained after splitting of point at each site. (b) Plaquette becomes an octagon after point splitting. Action of $\operatorname{Tr} U_{o}$ increases $n_{i} / m$ on each link on the octagon by \pm 1 .

\section{Splitting of the point}

In order to get a complete basis, we 'split' each site into two and insert a new link in between [2] thereby replacing each 4-vertex with two 3-vertices. This can be done in three different ways

\footnotetext{
${ }^{1}$ This is just the basic mandelstam relation connecting $\mathrm{SU}(2)$ Wilson loops recast in terms of Schwinger bosons.
} 
at each vertex as shown in figure 1(b),(c) and (d). In this paper, we choose the splitting scheme (d) at each site. This process of modifying the lattice is called splitting of the point and the resulting lattice is called a ps-lattice. Link operators and electric fields are introduced on the new link along with Gauss law constraints on each 3-vertex. Equivalently, Schwinger bosons satisfying link constraints are introduced at the ends of the new links. The Gauss law at one of the two new vertices can be used to fix the link operator of the new link to 1 . This gives back the original lattice. Therefore, ps-lattice describes the same physics as the original lattice. At any 3-vertex, there are 3 prepotentials (see figure 2(b)) denoted as $a_{i, \alpha}^{\dagger}, a_{i, \alpha}^{\dagger}, a_{3, \alpha}^{\dagger}$ where $i=1,2$ according to whether the vertex contains x-links or y-links. On every 3 -vertex, $\left(a_{i}^{\dagger} \cdot \tilde{a}_{\bar{i}}^{\dagger}\right),\left(a_{i}^{\dagger} \cdot \tilde{a}_{3}^{\dagger}\right),\left(a_{3}^{\dagger} \cdot \tilde{a}_{i}^{\dagger}\right)$ are locally gauge invariant and independent. So, a complete, orthonormal, gauge invariant local basis at a site is given by:

$$
\left|l_{i \bar{i}}, l_{\bar{i} \overline{3}}, l_{3 i}\right\rangle=\frac{\left(a_{i}^{\dagger} \cdot \tilde{a}_{\bar{i}}^{\dagger}\right)^{l_{i \bar{i}}}\left(a_{\bar{i}}^{\dagger} \cdot \tilde{a}_{3}^{\dagger}\right)^{l_{\bar{i} 3}}\left(a_{3}^{\dagger} \cdot \tilde{a}_{i}^{\dagger}\right)^{l_{3 i}}}{\sqrt{\left(l_{i \bar{i}}+l_{i 3}+l_{\bar{i} 3}+1\right) !\left(l_{i \bar{i}}\right) !\left(l_{3 i}\right) !\left(l_{\bar{i} 3}\right) !}}|0\rangle
$$

In (3.1), $l_{i \bar{i}}, l_{\bar{i}}, l_{3 i}$ are positive integers. Above states can also be labelled by the eigenvalues of the number operators $\hat{N}_{i}=a_{i}^{\dagger} \cdot a_{i}, \hat{N}_{2}=a_{\bar{i}}^{\dagger} \cdot a_{\bar{i}}, \hat{N}_{3}=a_{3}^{\dagger} \cdot a_{3}$. I.e, $\left|l_{i \bar{i}}, l_{\bar{i}}, l_{3 i}\right\rangle \equiv\left|n_{i}, n_{\bar{i}}, m \equiv n_{3}\right\rangle$ where $n_{i}=l_{i \bar{i}}+l_{3 i}, n_{\bar{i}}=l_{i \bar{i}}+l_{\bar{i} 3}, m \equiv n_{3}=l_{i 3}+l_{\bar{i} 3}$. The inverse relations are $l_{i \bar{i}}=n_{i}+n_{\bar{i}}-n_{3}, l_{\bar{i} 3}=n_{\bar{i}}+n_{3}-$ $n_{i}, l_{3 i}=n_{3}+n_{i}-n_{\bar{i}}$. Since, $E^{2}=\frac{\hat{N}}{2}\left(\frac{\hat{N}}{2}+1\right)$, number of prepotentials on a link is a measure of the electric flux through the link. The $n_{i}, m$ quantum numbers of the links meeting at a 3 vertex satisfies triangle inequalities. For eg, at a 3-vertex: $\left|n_{i}-n_{\bar{i}}\right| \leq m \leq n_{i}+n_{\bar{i}}$ In other words, $m(\vec{x})$ is limited by the value of $n_{i}(\vec{x})$ which in turn can take values freely between 0 and $\infty$. Alternatively, $l_{i \bar{i}}, l_{\bar{i} 3}, l_{3 i}$ at a site can be varied independently. The physical state space can be described equivalently in terms of (i) $l_{i j}$ at each site and the link constraints on every link implying a collection of overlapping, closed electric flux loops or (ii) $n_{i}$ and $m$ on the links satisfying local triangle inequalities at each 3-vertex. This equivalence can be realised only on the ps-lattice where the coordination number of any vertex is always three.

The Hamiltonian on the ps-lattice in the prepotential representation is given by

$$
H=g^{2} \sum_{\vec{x}} \frac{\hat{N}_{i}(\vec{x})}{2}\left(\frac{\hat{N}_{i}(\vec{x})}{2}+1\right)+\frac{2}{g^{2} a^{2}} \sum_{o c t}\left[2-\operatorname{Tr} U_{o}\right]
$$

Above, $\vec{x}$ denotes 3 -vertices, $i=1,2$ according as the 3 -vertex under consideration and $\operatorname{Tr} U_{o}$ is the product of the link operators in the octagon (see figure 2(b)) and is given by the Trace of product of $8 P(i j)$ matrices associated with the 8 vertices in the octagon and is given by:

$$
\hat{P}(i j)=\left(\begin{array}{cc}
\frac{1}{\sqrt{\hat{N}_{i}\left(\hat{N}_{j}+1\right)}}\left(a_{i}^{\dagger} \cdot \tilde{a}_{j}^{\dagger}\right) & \frac{1}{\sqrt{\hat{N}_{i}\left(\hat{N}_{j}+1\right)}}\left(a_{i}^{\dagger} \cdot a_{j}\right) \\
\frac{1}{\sqrt{\left(\hat{N}_{i}+2\right)\left(\hat{N}_{j}+1\right)}}\left(a_{i} \cdot a_{j}^{\dagger}\right) & \frac{1}{\sqrt{\left(\hat{N}_{i}+2\right)\left(\hat{N}_{j}+1\right)}}\left(\tilde{a}_{i} \cdot a_{j}\right)
\end{array}\right)
$$

At each 3-vertex along the octagon, there are two links which lie on the octagon. These links are labelled by $i$ and $j$ in (3.3). $\hat{P}(i j)_{11} / \hat{P}(i j)_{22}$ increases/decreases both $n_{i}, n_{j}$ by $1, \hat{P}(i j)_{12}$ increases $n_{i}$ but decreases $n_{j}$ and $\hat{P}(i j)_{21}$ increases $n_{j}$ but decreases $n_{i}$. $\operatorname{Tr} U_{o}$ has $2^{8}$ terms. Each term changes $n_{i} / m$ at the eight links of the octagon by \pm 1 thereby exhausting the $2^{8}$ possible ways to do it.

In order to construct a gauge invariant path integral to study the dynamics, we define phase operators [6] $e^{i \hat{\phi}_{i}}, e^{i \hat{\chi}}$, conjugate to $n_{i}, m$. For this, we first extend the Hilbert space by increasing 
the domain of $n_{i}, m$ to $(-\infty, \infty)$. Phase operators are defined such that they satisfy the commutation relations: $\left[n_{i}, e^{i \hat{\phi}_{i}}\right]=e^{i \hat{\phi}_{i}}\left[m, e^{i \hat{\chi}}\right]=e^{i \hat{\chi}} . \quad e^{i \hat{\phi}_{i}} / e^{-i \hat{\phi}_{i}}, e^{i \hat{\chi}} / e^{-i \hat{\chi}}$ act as step operators on the number states increasing/decreasing $n_{i}, m$ by 1 . We then define an eigenbasis of the phase operator $e^{i \hat{\phi}_{i}}, e^{i \hat{\chi}}$ : $e^{i \hat{\phi}_{i}}\left|\phi_{1}, \phi_{2}, \chi\right\rangle=e^{i \phi_{i}}\left|\phi_{1}, \phi_{2}, \chi\right\rangle e^{i \hat{\chi}}\left|\phi_{1}, \phi_{2}, \chi\right\rangle=e^{i \chi}\left|\phi_{1}, \phi_{2}, \chi\right\rangle$ The gauge invariant path integral is then constructed as the probability amplitude to go from the state $\left|\phi_{1}, \phi_{2}, \chi\right\rangle$ to $\left|\bar{\phi}_{1}, \bar{\phi}_{2}, \bar{\chi}\right\rangle$ in time $\mathrm{t}$ by the conventional time slicing method. The domain of $n_{i}, m$ is then restricted to positive values of $n_{i}, m$ within the path integral to get back to the original Hilbert space. The Euclidean path integral thereby constructed is given by :

$$
Z=\int D \phi_{1} D \phi_{2} D \chi \sum_{n_{1}, n_{2}, m}^{\prime} e^{-\int d t\left(g^{2} \sum_{\bar{x}}\left(\frac{n_{i}}{2}\left[\frac{n_{i}}{2}+1\right]\right)+\frac{2}{g^{2} a^{2}} \sum_{o c t}\left[2-\operatorname{Tr}\left\{P_{o c t}\right\}\right]+\sum_{\vec{x}}\left\{i n_{1} \dot{\phi}_{1}+i n_{2} \dot{\phi}_{2}+i m \dot{\chi}\right\}\right)}
$$

We associate a matrix $S\left(n_{i}, m, n_{\bar{i}}\right)$ to each vertex and a matrix $Q\left(\phi_{i}\right)$ to each link in the octagon.

$S\left(n_{i}, m, n_{\bar{i}}\right)=\left(\begin{array}{l}\sqrt{\frac{\left(n_{i}+n_{i}+m+3\right)\left(n_{i}-n_{\bar{i}}+m+1\right)}{4(m+1)\left(n_{i}+1\right)}} \sqrt{\frac{\left(n_{\bar{i}}-n_{i}+m+1\right)\left(n_{\bar{i}}+n_{i}-m+1\right)}{4(m+1)\left(n_{i}+1\right)}} \\ \sqrt{\frac{\left(n_{\bar{i}}-n_{i}+m+1\right)\left(n_{\bar{i}}+n_{i}-m+1\right)}{4(m+1)\left(n_{i}+1\right)}} \sqrt{\frac{\left(n_{i}+n_{\bar{i}}+m+3\right)\left(n_{i}-n_{\bar{i}}+m+1\right)}{4(m+1)\left(n_{i}+1\right)}}\end{array}\right) ; Q\left(\phi_{i}\right)=\left(\begin{array}{cc}e^{i \phi_{i}} & 0 \\ 0 & e^{-i \phi_{i}}\end{array}\right)$

$P_{\text {oct }}$ in eqn. (3.4) is the ordered product of the $S$ and $Q$ matrices included within the octagon. In (3.4), the summation is over positive $n_{1}, n_{2}, m$ satisfying triangle inequality at each 3 -vertex.

\section{The weak coupling limit}

We now make a useful ansatz to study the weak coupling limit by constructing a weak coupling expansion. Since the magnetic term is strictly positive and its lowest value occurs when $U(\vec{x})$ reach $\mathbf{1}$, this in the gauge invariant configuration space leads to $P_{o c t} \rightarrow 1$ and $S \rightarrow \mathbf{1}$. Furthermore, all the integer fields $n_{i}, m$ are always positive and hence $\left\langle n_{i}\right\rangle$ and $\langle m\rangle$ are non zero. If we assume $\left\langle n_{i}\right\rangle=N$ and insist that $S \sim 1$, we note that $\langle m\rangle=2 N$ and $\mathrm{N}$ is large with $\phi_{i}, \chi \rightarrow 0$, as the only solution. We rescale ${ }^{2} \phi_{i}, \chi \rightarrow \frac{1}{2} g a \phi_{i}, \frac{1}{2} g a \chi$ and make the substitution, $n_{i} \equiv N+\tilde{n}_{i}, m \equiv 2 N+\tilde{m}$. $\operatorname{Tr} P_{\text {oct }}$ consists of $2^{8}$ terms corresponding to all possible fluctuations generated by the plaquette term along the octagon(see figure 2(b)). In each of the terms, the off diagonal terms come in pairs. This is due to the link constraint (2.2). The off-diagonal terms of $S$ are at least of the order of $\frac{1}{2 \sqrt{N}}$. Therefore, the terms can be classified according to the number of off diagonal terms occurring. Such a classification corresponds to an expansion in $\frac{1}{N}$. The leading term $\frac{1}{N^{0}}$ is the term which does not contain any off diagonal terms and corresponds to the term which either increases(+) or decreases(-) the field $n_{i}, m$ at every link along the octagon. Every other term consists of several flips $+\rightarrow-$ or $-\rightarrow+$ along the octagon and each flip brings in a $\frac{1}{2 \sqrt{N}}$. Consistency demands that each term have even number of flips. The second leading term has two flips and hence is of the order of $\frac{1}{4 N}$. The next term $\frac{1}{N^{2}}$ consists of four such flips. After a straight forward tedious calculation [2], we get

$$
\operatorname{Tr} P_{o c t} \approx 2-\left(\frac{1}{4 N^{2}} \tilde{m}^{2}+V\left(\phi_{1}, \phi_{2}, \chi\right)\right)
$$

\footnotetext{
${ }^{2}$ This gives $\phi_{i}^{2}, \chi^{2}$ the dimensions of energy and $\frac{1}{2}$ factor is for later convenience.
} 


$$
\begin{aligned}
V\left(\phi_{1}, \phi_{2}, \chi\right)=\frac{g^{2} a^{2}}{4}\{ & {\left[\left(\Delta_{1}\left(\phi_{2}-\frac{1}{2} \Delta_{2} \chi\right)-\Delta_{2}\left(\phi_{1}+\frac{1}{2} \Delta_{1} \chi\right)\right)\right]^{2}+\frac{1}{N}\left[16\left[\left(\phi_{1}+\frac{1}{2} \Delta_{1} \chi\right)^{2}+\left(\phi_{2}-\frac{1}{2} \Delta_{2} \chi\right)^{2}+\chi^{2}\right]\right.} \\
& \left.\left.-\left[\Delta_{1}\left(\phi_{2}-\frac{1}{2} \Delta_{2} \chi\right)-\Delta_{2}\left(\phi_{1}+\frac{1}{2} \Delta_{1} \chi\right)+\Delta_{1} \Delta_{2} \chi\right]^{2}-\left(\Delta_{1} \Delta_{2} \chi\right)^{2}\right]\right\}
\end{aligned}
$$

The path integral becomes:

$$
Z=\int_{-\frac{\pi}{g}}^{\frac{\pi}{g}} D \phi_{1} D \phi_{2} D \chi \sum_{\substack{\tilde{n}_{1}=-N \\ \tilde{n}_{2}=-N}}^{\infty} \sum_{\substack{2 \\ n_{2}}}^{M_{2}} e^{\left.-\int d t \sum_{\text {sites }}\left[\frac{g^{2}}{2}\left(\tilde{n}_{1}^{2}+\tilde{n}_{2}^{2}+2 N^{2}\right)+\frac{1}{2 g^{2} a^{2} N^{2}} \tilde{m}^{2}+i \tilde{n}_{i} \frac{1}{2} a g \dot{\phi}_{i}+i \tilde{m} \frac{1}{2} a g \dot{\chi}+\frac{1}{2 g^{2}} V\left(\phi_{1}, \phi_{2}, \chi\right)\right]\right]}
$$

Above, $M_{1}=\min \left(\tilde{n}_{1}+\tilde{n}_{\overline{1}}, \tilde{n}_{2}+\tilde{n}_{\overline{2}}\right), M_{2}=\max \left(2 N-\left|\tilde{n}_{1}-\tilde{n}_{\overline{1}}\right|, 2 N-\left|\tilde{n}_{2}-\tilde{n}_{\overline{2}}\right|\right)$. This is due to the fact that $\tilde{m}$ is limited by the triangle inequality. In the weak coupling limit, $M_{1} \approx 0, M_{2} \approx 2 N$. Therefore, triangle inequality becomes irrelevant upto the leading term in the weak coupling limit.

Using Euler-Maclaurin formula to convert the summation over $\tilde{n}$ and $\tilde{m}$ to integration and performing the integral, we get upto an irrelevant constant $\mathrm{C}$ :

$$
\begin{gathered}
Z=C \int D \phi_{1} D \phi_{2} D \chi e^{-W\left[N, \phi_{i}, \dot{\phi}_{i}, \chi, \dot{\chi}\right]} \\
W\left[N, \phi_{i}, \dot{\phi}_{i}, \chi, \dot{\chi}\right]=\int d t \sum_{\text {sites }}\left[\frac{a^{2}}{4}\left(\dot{\phi}_{1}{ }^{2}+\dot{\phi}_{2}{ }^{2}\right)+\frac{g^{2} a^{2}}{4} \frac{\dot{\chi}^{2}}{2 /\left(g^{2} a^{2} N^{2}\right)}+\frac{1}{2 g^{2}} V\left(\phi_{1}, \phi_{2}, \chi\right)\right]
\end{gathered}
$$

We now make the following transformation,

$$
\phi_{i}=\frac{1}{\sqrt{-\Delta^{2}}}\left(\Delta_{i} \eta+\varepsilon_{i j} \delta_{j} \psi\right)+\frac{1}{2} \varepsilon_{j i} \Delta_{j} \chi
$$

In (4.6), $\Delta^{2}$ is the lattice laplacian and $\delta_{j}$ is the backward difference operator defined as $\Delta^{2}=$ $\sum_{i}\left(\Delta_{i}-\delta_{i}\right) ; \delta_{j} f(\vec{x})=f(\vec{x})-f(\vec{x}-\hat{j})$. Ignoring constant terms, the path integral becomes:

$$
\begin{gathered}
Z=\int D \psi D \eta D \chi e^{-\int d t \sum_{\text {sites }}\left[\frac{a^{2}}{4}\left(\dot{\eta}^{2}+\dot{\psi}^{2}\right)+\frac{1}{8} g^{4} a^{4} N^{2} \dot{\chi}^{2}+\frac{a^{2}}{4}\left(\frac{1}{4}\left(\left(\Delta_{1} \dot{\chi}\right)^{2}+\left(\Delta_{2} \dot{\chi}\right)^{2}\right)+\dot{\chi} \frac{1}{\sqrt{-\Delta^{2}}}\left[\left(\delta_{1} \Delta_{1}-\delta_{2} \Delta_{2}\right) \dot{\eta}+2 \delta_{1} \delta_{2} \dot{\psi}\right]\right)+\frac{2}{g^{2} a^{2}} V(\psi, \eta, \chi)\right]} \\
V(\psi, \eta, \chi)=\frac{1}{2} g^{2} a^{2}\left\{\frac{1}{4}(\Delta \psi)^{2}+\frac{1}{N}\left[4\left(\eta^{2}+\psi^{2}+\chi^{2}\right)-(\Delta \psi)^{2}-2\left(\Delta_{1} \Delta_{2} \chi\right)^{2}+\Delta^{2} \chi \frac{\Delta_{1} \Delta_{2}}{\sqrt{-\Delta^{2}}} \chi\right]\right\}
\end{gathered}
$$

Above, $(\Delta \psi)^{2} \equiv\left(\Delta_{1} \psi\right)^{2}+\left(\Delta_{2} \psi\right)^{2}$. The continuum limit is now taken by making

$$
\frac{16}{N}=M^{2} a^{2}
$$

where $M$ is the mass in the continuum and $a$ is the lattice constant. Consequently, the euclidean inverse propagators in the energy-momentum space to the leading order are

$$
\begin{aligned}
& \psi: p_{0}^{2}+M^{2}+\vec{p}^{2}+O\left(a^{2}\right) \\
& \eta: p_{0}^{2}+M^{2}+O\left(a^{4}\right) \\
& \chi: \# a^{2} \vec{p}^{2} p_{0}^{2}+M^{2}+O\left(a^{4}\right)
\end{aligned}
$$

Above, \# denotes a real positive constant. Therefore, $\psi$ is a relativistic particle with mass $M$ and $\chi$ do not propagate. $\eta$ may propagate due to higher order corrections. 


\section{Summary and Future directions}

A complete gauge invariant electric basis of the Hilbert space can be constructed [3] on a square lattice by solving the Gauss law at each site of the lattice. However, in such a description dynamics is complicated and involves higher Wigner symbols. In order to simplify the dynamics, we recast the theory in terms of Schwinger Bosons (prepotentials) which transform under the fundamental representation of the gauge group. But, the basis thus constructed by making singlets out of the prepotentials at each site turns out to be over complete. In order to have a complete basis, we construct a point split lattice. Point splitting enables us to analyze a theory of interacting closed loops in terms of local quantum numbers satisfying triangle inequalities. Dynamics was studied by constructing a phase space path integral over positive integers $n_{1}, n_{2}, m$ and the continuous phases $\phi_{1}, \phi_{2}, \chi$. Such a path inegral can be used for numerical simulations. Alternatively, one could work within the Hamiltonian formulation to study the dynamics by other methods like tensor networks, or cold atom simulations.

On inclusion of fermions, the Gauss law at each site is modified. It leads to a modified point splitting scheme as well as new singlets involving fermions and thereby a modified ps-basis. The prepotential representation of SU(3) gauge theory is already available in the literature [5] and there doesn't seem to be any serious hindrance in the extension of the point splitting scheme to SU(3) and higher dimensionsional lattices. The dispersion relations (4.10) which was deduced upto the lowest order in weak coupling expansion seems to imply that the lowest excitation is a non-degenerate scalar which is consistent with the existing literature [7]. Lorentz covariance as well as the interactions in the higher orders need to be investigated.

\section{References}

[1] K. G. Wilson, Phys. Rev. D 10 (1974) 2445. A. M. Polyakov, Nucl. Phys. B 164 (1979) 171. S. Mandelstam, Phys. Rev. D 19 (1979) 2391; R. Gambini, Jorge Pullin, Loops, Knots, Gauge Theories and Quantum Gravity (Cambridge University Press, 2000); Manu Mathur, T. P. Sreeraj, Phys. Rev. D 92,(2015) 125018; Phys. Lett. B 749 (2015) 137; Phys. Rev. D 94 (2016) 085029 and references therein.

[2] Ramesh Anishetty and Sreeraj T P, Phys Rev D 97, 074511 (2018); R. Anishetty and I. Raychowdhury, Phys. Rev. D 90, no. 11, 114503 (2014)

[3] R. Anishetty and H. S. Sharatchandra, Phys. Rev. Lett. 65, 813 (1990); H. S. Sharatchandra, Nucl. Phys. B 196 (1982) 62; R. Anishetty, Phys. Rev. D 44, 1895 (1991).

[4] J. Kogut, L. Susskind, Phys. Rev. D 11 (1975) 395.

[5] M. Mathur, J. Phys. A 38, 10015 (2005) Nucl. Phys. B 779, 32 (2007); Phys. Lett. B 640, 292 (2006); M. Mathur, I. Raychowdhury and R. Anishetty, J. Math. Phys. 51, 093504 (2010); R. Anishetty, M. Mathur and I. Raychowdhury, J. Phys. A 43, 035403 (2010), J. Math. Phys. 50, 053503 (2009); M. Mathur and I. Raychowdhury, J. Phys. A 44, 035203 (2011)

[6] P. Carruthers, M.M. Nieto, Rev. Mod. Phys 40(1968) 411.

[7] D. Karabali, V. P. Nair, Nuclear Physics B 464 (1996) 135-152; D. Karabali , C. Kim, V. P. Nair, Nucl. Phys. B 524 (1998) 661-694; Phys. Rev. D, 64, 025011 (2001); Peter Orland, Phys Rev D 71, 054503 (2005); Michael J Teper, Phys. Rev. D 59 (1998) 014512. 